\title{
Quasielastic contribution to antineutrino-nucleus scattering
}

\author{
V. Pandey, ${ }^{*}$ N. Jachowicz, ${ }^{\dagger}$ J. Ryckebusch, T. Van Cuyck, and W. Cosyn \\ Department of Physics and Astronomy, Ghent University, Proeftuinstraat 86, B-9000 Gent, Belgium
}

(Received 25 October 2013; published 4 February 2014)

\begin{abstract}
We report on a calculation of cross sections for charged-current quasielastic antineutrino scattering off ${ }^{12} \mathrm{C}$ in the energy range of interest for the MiniBooNE experiment. We adopt the impulse approximation (IA) and use the nonrelativistic continuum random phase approximation (CRPA) to model the nuclear dynamics. An effective nucleon-nucleon interaction of the Skyrme type is used. We compare our results with the recent MiniBooNE antineutrino cross-section data and confront them with alternate calculations. The CRPA predictions reproduce the gross features of the shape of the measured double-differential cross sections. The CRPA cross sections are typically larger than those of other reported IA calculations but tend to underestimate the magnitude of the MiniBooNE data. We observe that an enhancement of the nucleon axial mass in CRPA calculations is an effective way of improving on the description of the shape and magnitude of the double-differential cross sections. The rescaling of $M_{A}$ is illustrated to affect the shape of the double-differential cross sections differently than multinucleon effects beyond the IA.
\end{abstract}

DOI: 10.1103/PhysRevC.89.024601

PACS number(s): 25.30.Pt, 13.15.+g, 24.10.Cn, 21.60.Jz

\section{INTRODUCTION}

Recent times have been marked by a substantial increase in the amount of data for (anti)neutrino-nucleus interactions at intermediate energies. Recently, the MiniBooNE collaboration has published their first charged-current quasielastic (CCQE) antineutrino-nucleus scattering cross-section measurements [1]. The underlying reaction process of CCQE with antineutrino beams is $\bar{v}_{\mu}+p \rightarrow \mu^{+}+n$ on bound protons. Antineutrino-nucleus $(\bar{v} A)$ cross sections are less well measured than their neutrino counterparts, mainly because of higher background contributions and smaller statistics [2]. MiniBooNE has also published cross sections for CCQE neutrino $\left(v_{\mu}+n \rightarrow \mu^{-}+p\right)$ [3] and neutral-current quasielastic (NCQE) neutrino $\left(v_{\mu}+N \rightarrow v_{\mu}+N\right)$ [4] processes. Several other collaborations have been contributing to the increase of the neutrino-nucleus cross-section database in recent times. For example, T2K has released inclusive $\mathrm{CC}$ neutrino [5] data, whereas MINERvA presented CC neutrino [6] and antineutrino [7] cross-section results.

The modeling of $v A$ and $\bar{v} A$ scattering data poses some real challenges. In contrast to electron-nucleus scattering data for which the initial electron energy is exactly known, the $\nu A$ and $\bar{v} A$ data are $v(\bar{v})$-flux integrated [8]. Despite the enormous improvements in the experimental and theoretical understanding of (anti)neutrino-nucleus interactions in the few $\mathrm{GeV}$ region, the current experimental precision is of the order of $20 \%-30 \%$ and the underlying processes on bound nucleons are not fully understood [2,8-10]. Theoretical predictions for MiniBooNE's $\bar{v}_{\mu}+{ }^{12} \mathrm{C}$ measurements are reported in Refs. [11-15]. References [11,12] adopt a rather basic nuclear-structure model which can not be expected to capture the complexity of the nuclear dynamics at low nuclear excitation energies. Reference [13] starts from a relativistic

\footnotetext{
*Vishvas.Pandey@UGent.be

†Natalie.Jachowicz@UGent.be
}

mean-field model for the bound and scattering states. The approach in Ref. [14] is based on superscaling approximation and Ref. [15] adopts a relativistic Green's function model. Reference [11] computes nuclear response functions with a local Fermi-gas model in the random phase approximation (RPA) and incorporates multinucleon effects exclusively in the spin-isospin channels. Reference [12] starts from a local Fermi-gas description of the nucleus and includes RPA correlations and multinucleon effects. Both calculations for the $\bar{v}_{\mu}+{ }^{12} \mathrm{C}$ responses stress the importance of multinucleon mechanisms at MiniBooNE kinematics, and adopt a value for the axial mass $\left(M_{A} \approx 1 \mathrm{GeV}\right)$ in a dipole parametrization of the axial form factor, which is consistent with the one used to model the QE contribution to $v_{\mu}+{ }^{12} \mathrm{C}$ [16-19]. The multinucleon mechanisms account for mechanisms in the $W$-nucleus coupling beyond the impulse approximation (IA). In the IA, the $W$-nucleus coupling is approximated as a sum of one-body $W$-nucleon couplings. Effects beyond the IA introduce some uncertainties in the calculations, particularly for finite nuclei as a consistent treatment of the multinucleon electroweak currents is extremely challenging. According to a recent study of neutrino scattering off the deuteron, the effect of two-body currents (excluding pion production channels) is smaller than $10 \%$ [20].

In this work, we adopt the IA for modeling the electroweaknucleus coupling and use a more sophisticated model for describing the structure of the initial and final nuclei. In our approach to investigate MiniBooNE's CCQE $\bar{v}_{\mu}+{ }^{12} \mathrm{C}$ results, we model the nuclear dynamics starting from the mean-field (MF) description and introduce long-range correlations by means of a nonrelativistic continuum RPA (CRPA) framework. Thereby, we use Green's functions (or propagators) to solve the CRPA equations and an effective Skyrme nucleon-nucleon residual interaction. The model takes into account one-particle one-hole (1p-1h) excitations out of a correlated nuclear ground state. In the CRPA, the effects of final-state interactions of the ejected nucleons with the residual nucleus are implemented. Thereby, one accounts for both distortions on the ejected 
nucleon waves and rescatterings with the residual $A-1$ nucleons. For example, rescattering effects $\bar{v}_{\mu}+p+(A-1) \rightarrow$ $\mu^{+}+n+(A-1) \rightarrow \mu^{+}+n^{\prime}+(A-1)^{\prime}$ are included. In CRPA, the strength of the rescatterings is regulated with the residual nucleon-nucleon force. In the results section, we focus on the influence of RPA correlations on the computed antineutrino responses for the MiniBooNE kinematics. The CRPA formalism does not contain relativistic corrections in its description of the nuclear dynamics. In Refs. [17,21,22], one proposes to correct the energy transfer $\omega$ to account for relativistic effects in nonrelativistic Fermi-gas calculations. These methods, however, can not be readily applied to the CRPA framework, as the computed response scales with the asymptotic nucleon kinetic energies in a complicated fashion. It is worth mentioning that MiniBooNE's antineutrino flux distribution is shifted to lower energies compared to the neutrino one. Therefore, it can be anticipated that the $\bar{v}_{\mu}+{ }^{12} \mathrm{C}$ responses are subject to smaller relativistic corrections than the $v_{\mu}+{ }^{12} \mathrm{C}$ ones.

The paper is organized as follows. In Sec. II, we briefly describe the CRPA framework of our cross-section calculations. In Sec. III, we present numerical results of $\bar{v}_{\mu}+{ }^{12} \mathrm{C}$ cross sections and compare them with the MiniBooNE data and with other theoretical models. The conclusions are given in Sec. IV.

\section{FORMALISM}

In this work, we focus on the inclusive CCQE antineutrino nuclear reaction

$$
\bar{v}_{\mu}+{ }^{12} \mathrm{C} \rightarrow \mu^{+}+\mathrm{X}
$$

with no pion in the final state, a process which is referred to as QE-like [16,17,23]. We obtain nuclear responses with the CRPA method, which is described in details in Refs. [24,25]. This formalism has been successfully used in the description of exclusive photoinduced and electroinduced QE processes [26,27] and in inclusive neutrino scattering at supernova energies [24,25,28,29]. Here, the CRPA method is applied to antineutrino-nucleus interactions at intermediate energies. The CRPA framework includes all single-nucleon knockout channels and is therefore well suited to compute the quasielastic contribution to the inclusive (anti)neutrinonucleus responses. The CRPA framework is not suited to compute the contributions from alternate reaction mechanisms such as multinucleon knockout.

We summarize the basis ingredients of the model. An effective Skyrme two-body interaction (more specifically, the SkE2 parametrization [26]) is used to construct a mean-field (MF) potential. The bound and continuum single-nucleon wave functions can be obtained as the solutions to the corresponding Schrödinger equation. The long-range correlations between the nucleons are introduced through the RPA which describes an excited nuclear state with a nucleon in the energy continuum of the MF potential as the coherent superposition of particle-hole $\left(\mathrm{ph}^{-1}\right)$ and hole-particle $\left(\mathrm{hp}^{-1}\right)$ excitations out of a correlated ground state, which has $0 \mathrm{p}-0 \mathrm{~h}$ and $2 \mathrm{p}-2 \mathrm{~h}$ components

$$
\left|\Psi_{\mathrm{RPA}}^{C}\right\rangle=\sum_{C^{\prime}}\left\{X_{C, C^{\prime}}\left|\mathrm{ph}^{-1}\right\rangle-\mathrm{Y}_{\mathrm{C}, \mathrm{C}^{\prime}}\left|\mathrm{hp}^{-1}\right\rangle\right\} .
$$

Here, $C^{\prime}$ stands for a combination of all quantum numbers of a hole and particle state. Green's function theory allows one to treat the single-particle energy continuum exactly [26]. In computing the response of the nucleus to an external electroweak probe, a key quantity is the RPA polarization propagator which can be obtained as a solution to the following iterative equation:

$$
\begin{aligned}
\Pi^{(\mathrm{RPA})}\left(x_{1}, x_{2} ; E_{x}\right)= & \Pi^{(0)}\left(x_{1}, x_{2} ; E_{x}\right)+\frac{1}{\hbar} \int d x d x^{\prime} \Pi^{0} \\
& \times\left(x_{1}, x ; E_{x}\right) \tilde{V}\left(x, x^{\prime}\right) \Pi^{(\mathrm{RPA})}\left(x^{\prime}, x_{2} ; E_{x}\right),
\end{aligned}
$$

where $E_{x}$ is the excitation energy of the target nucleus and $x$ is a shorthand notation for the combination of the spatial, spin, and isospin coordinates. Further, $\Pi^{(0)}$ denotes the MF contribution to the polarization propagator and $\tilde{V}$ is the antisymmetrized residual interaction. The MF responses can be computed by neglecting the second term in the above equation. The second term accounts for the multiple-scattering events after the initial electroweak excitation of a nucleon from a bound into a continuum state. In the MF approach, only direct nucleon knockout is included and the sole implemented final-state interaction (FSI) effect is the distortion of the ejected-nucleon waves in the real MF potential of the residual nucleons.

In terms of the experimentally measured quantities (outgoing muon kinetic energy $T_{\mu}$ and cosine of the muon scattering angle $\cos \theta_{\mu}$ ), the twofold differential cross section for CC (anti)neutrino-nucleus scattering is given by

$$
\begin{aligned}
\left(\frac{d^{2} \sigma}{d T_{\mu} d \cos \theta_{\mu}}\right)_{\nu, \bar{v}}= & G_{F}^{2} \cos ^{2} \theta_{c}\left(\frac{2}{2 J_{i}+1}\right) \varepsilon_{\mu}^{2} \tilde{k}_{\mu} F\left(Z^{\prime}, \varepsilon_{\mu}\right) \\
& \times\left[\sum_{J=0}^{\infty} \sigma_{\mathrm{CL}}^{J}+\sum_{J=1}^{\infty} \sigma_{T}^{J}\right]
\end{aligned}
$$

where $G_{F}$ is the weak interaction coupling constant and $\theta_{c}$ is the Cabibbo angle. Further, $\tilde{k}_{\mu}=k_{\mu} / \varepsilon_{\mu}$ with $k_{\mu}\left(\varepsilon_{\mu}\right)$ is the momentum (energy) of the final lepton. The Fermi function $F\left(Z^{\prime}, \varepsilon_{\mu}\right)$ is introduced in order to take into account the Coulomb interaction between the outgoing lepton and the residual nucleus which has a proton number $Z^{\prime}$. In order to compute the differential cross sections, we rely on a multipole expansion of the weak transition operators and in Eq. (4) the $\sigma_{\mathrm{CL}}^{J}$ and $\sigma_{T}^{J}$ are the Coulomb longitudinal and the transverse contributions for a given multipolarity $J$ :

$$
\begin{aligned}
\sigma_{\mathrm{CL}}^{J}= & v^{\mathcal{M}}\left|\left\langle J_{f}|| \widehat{\mathcal{M}}_{J}(|\vec{q}|)|| J_{i}\right\rangle\right|^{2}+v^{\mathcal{L}}\left|\left\langle J_{f}|| \widehat{\mathcal{L}}_{J}(|\vec{q}|)|| J_{i}\right\rangle\right|^{2} \\
& +2 v^{\mathcal{M} \mathcal{L}} \mathcal{R}\left[\left\langle J_{f}|| \widehat{\mathcal{L}}_{J}(|\vec{q}|)|| J_{i}\right\rangle\left\langle J_{f}|| \widehat{\mathcal{M}}_{J}(|\vec{q}|)|| J_{i}\right\rangle^{*}\right],
\end{aligned}
$$


(a) Bjorken $x_{B}$

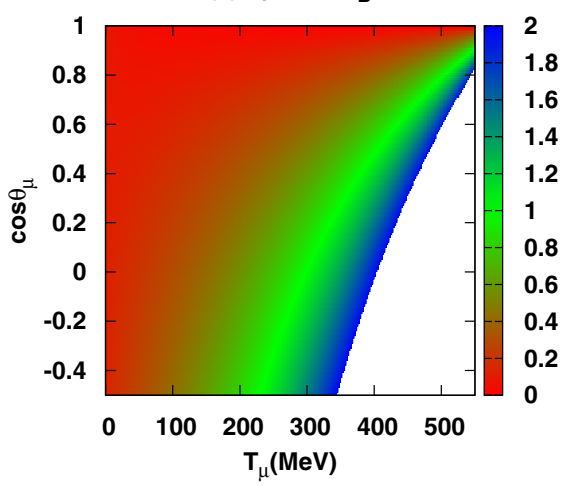

(b) Minimum missing momentum (MeV)

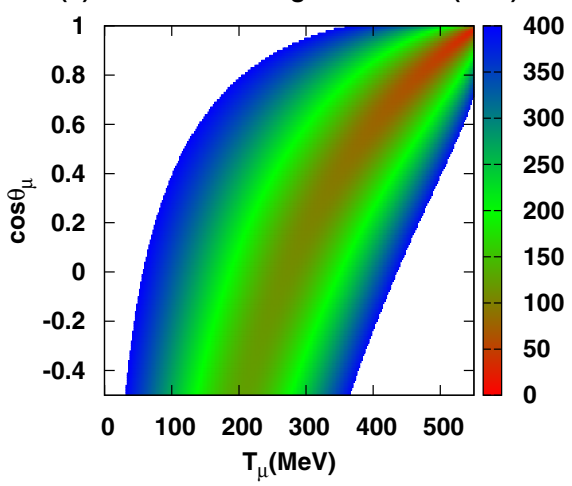

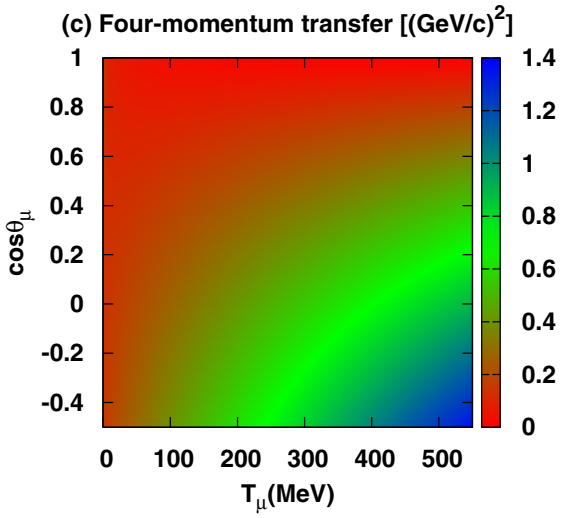

FIG. 1. (Color online) The kinematic variables (a) Bjorken $x_{B}$, (b) minimum $p_{\text {mis }}$, and (c) $Q^{2}$ as a function of $T_{\mu}$ and $\cos \theta_{\mu}$ at $E_{\bar{v}_{\mu}}=700 \mathrm{MeV}$. White regions correspond with values of the variables out of the specified ranges.

with

$$
\begin{aligned}
v^{\mathcal{M}} & =\left[1+\tilde{k}_{\mu} \cos \theta_{\mu}\right], \\
v^{\mathcal{L}} & =\left[1+\tilde{k}_{\mu} \cos \theta_{\mu}-\frac{2 \varepsilon_{i} \varepsilon_{\mu}}{|\vec{q}|^{2}} \tilde{k}_{\mu}^{2} \sin ^{2} \theta_{\mu}\right], \\
v^{\mathcal{M L}} & =\left[\frac{\omega}{|\vec{q}|}\left(1+\tilde{k}_{\mu} \cos \theta_{\mu}\right)+\frac{m_{\mu}^{2}}{\varepsilon_{\mu}|\vec{q}|}\right],
\end{aligned}
$$

and

$$
\begin{aligned}
\sigma_{T}^{J}= & v^{T}\left[\left|\left\langle J_{f} \| \widehat{\mathcal{J}}_{J}^{\mathrm{mag}}(|\vec{q}|)|| J_{i}\right\rangle\right|^{2}+\left|\left\langle J_{f} \| \widehat{\mathcal{J}}_{J}^{e l}(|\vec{q}|)|| J_{i}\right\rangle\right|^{2}\right] \\
& \mp 2 v^{T T} \mathcal{R}\left[\left\langle J_{f}|| \widehat{\mathcal{J}}_{J}^{\mathrm{mag}}(|\vec{q}|)|| J_{i}\right\rangle\left\langle J_{f} \| \widehat{\mathcal{J}}_{J}^{e l}(|\vec{q}|)|| J_{i}\right\rangle^{*}\right],
\end{aligned}
$$

with

$$
\begin{aligned}
v^{T} & =\left[1-\tilde{k}_{\mu} \cos \theta_{\mu}+\frac{\varepsilon_{i} \varepsilon_{\mu}}{|\vec{q}|^{2}} \tilde{k}_{\mu}^{2} \sin ^{2} \theta_{\mu}\right], \\
v^{\mathrm{TT}} & =\left[\frac{\varepsilon_{i}+\varepsilon_{\mu}}{|\vec{q}|}\left(1-\tilde{k}_{\mu} \cos \theta_{\mu}\right)-\frac{m_{\mu}^{2}}{\varepsilon_{\mu}|\vec{q}|}\right] .
\end{aligned}
$$

Here, $Q^{2}=-q^{\mu} q_{\mu}$, with $q^{\mu}(\omega, \vec{q})$ the transferred fourmomentum carried by the $W$ boson. $\varepsilon_{i}$ is energy of the incoming neutrino and $m_{\mu}$ is the mass of the final lepton. The $\widehat{\mathcal{M}}_{J}, \widehat{\mathcal{L}}_{J}, \widehat{\mathcal{J}}_{J}^{e l}$, and $\widehat{\mathcal{J}}_{J}^{\text {mag }}$ denote the Coulomb, longitudinal, transverse electric, and transverse magnetic transition operators as defined in Refs. [24,25]. The $|\vec{q}|$ is the magnitude of the transferred three-momentum and $J_{i}\left(J_{f}\right)$ represents the total angular momentum of the initial (final) state of the nucleus. The difference between the neutrino and antineutrino CC cross sections stems from the sign assigned to the interference term in Eq. (6): positive for the neutrino and negative for the antineutrino beams.

As mentioned, in this work we adopt the IA. Now, we introduce a number of variables which allow one to assess the validity of this approximation for given kinematic settings. The Bjorken $x_{B}$ scaling variable is given by the invariant quantity

$$
x_{B}=\frac{A Q^{2}}{2 p_{A}^{\mu} q_{\mu}},
$$

where $p_{A}^{\mu}$ is the momentum of the target nucleus. Figure 1 displays $x_{B}$ as a function of the experimentally measured quantities $T_{\mu}$ and $\cos \theta_{\mu}$ for $E_{\bar{v}_{\mu}}=700 \mathrm{MeV}$. As shown in Fig. 2, MiniBooNE's $\bar{v}_{\mu}$ energy spectrum reaches its mean near $700 \mathrm{MeV}$. For $x_{B} \approx 1, \mathrm{QE}$ single-nucleon knockout is expected to dominate and IA calculations are expected to perform best. From Fig. 1 it is clear that at very forward $\theta_{\mu}$, one expects the bulk of the single-nucleon knockout strength at larger $T_{\mu}$. With increasing $\theta_{\mu}$, the QE single-nucleon knockout strength will shift to lower $T_{\mu}$. At kinematic conditions corresponding with both low $T_{\mu}$ and forward muon scattering angles, one could expect major contributions beyond the IA.

The $x_{B}$ is a model-independent kinematic variable. We now introduce a kinematic variable which is a highly relevant one for $\mathrm{QE}$ processes. In direct single-nucleon knockout reactions, the momentum of the initial bound nucleon (often referred as the missing momentum $p_{\text {mis }}$ ) is the scaling variable [30]. Indeed, in the plane-wave limit, the exclusive singlenucleon knockout cross sections are directly proportional to the momentum distribution of the bound nucleons in the target nucleus. Mean-field nucleons are characterized by a momentum distribution which is Gaussian-type and extends over a specific range $\left(0 \leqslant p_{\text {mis }} \lesssim 250 \mathrm{MeV}\right)$ [31]. Large

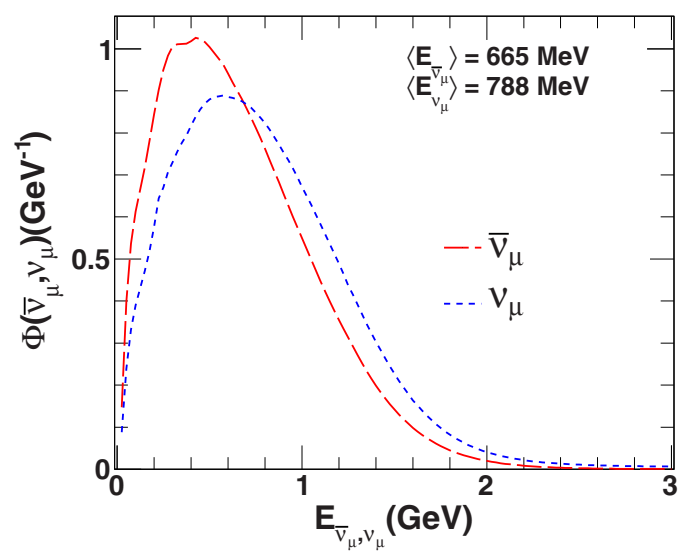

FIG. 2. (Color online) The MiniBooNE antineutrino and neutrino flux $[1,3]$ normalized to 1 . 
missing momenta necessarily lead to small single-nucleon knockout cross sections and/or substantial contributions from competing multinucleon processes. Imposing a $\mathrm{QE}$ reaction process ( $W^{-}+p$ with $A-1$ spectators), energy and momentum conservation in the laboratory frame can be expressed as

$$
M_{A}+\omega=E_{A-1}^{\star}+\sqrt{M_{n}^{2}+p_{n}^{2}}, \quad \vec{p}_{\text {mis }}+\vec{q}=\vec{p}_{n},
$$

where $\vec{p}_{n}$ is the three-momentum of the ejected neutron in the laboratory frame, $M_{n}$ is the neutron mass, and $M_{A}$ the mass of the target nucleus. The $E_{A-1}^{\star}$ is the total energy of the residual nucleus and includes contributions from recoil and excitation energy $E_{A-1}^{\star}=M_{A-1}+T_{A-1}+E_{\mathrm{exc}}^{\star}$. The $p_{\text {mis }}$ depends on $\theta_{p_{n} q}$, the angle between $\vec{q}$ and $\vec{p}_{n}$. For inclusive reactions as those considered in this work, the relative importance of the quasielastic contribution can be estimated with the aid of the minimum missing momentum: the minimum value of $p_{\text {mis }}$ as $\theta_{p_{n} q}$ varies between $0^{\circ}$ and $180^{\circ}$. In Fig. 1, we also display the minimum value of the missing momentum, denoted as $p_{\mathrm{mis}}^{\min }$ for a given incoming neutrino energy and $T_{A-1}+E_{\mathrm{exc}}^{\star}=25 \mathrm{MeV}$. As one moves along the $x_{B} \approx 1$ region, with increasing $\theta_{\mu}$ a shift to larger $p_{\mathrm{mis}}^{\min }$ is observed and larger multinucleon contributions can be expected [32]. The $\left(T_{\mu}, \cos \theta_{\mu}\right)$ kinematic settings with a minimum $p_{\text {mis }} \gtrsim 250 \mathrm{MeV}$ are prone to multinucleon corrections beyond the IA. For the sake of completeness, we also show a contour plot of the $W$ boson's virtuality. Kinematic regions with the lowest $Q^{2}$ exhibit the strongest sensitivity to collective nuclear structure mechanisms.

The wide range of values of $\left(x_{B}, p_{\mathrm{mis}}^{\min }, Q^{2}\right)$ probed in the MiniBooNE $\bar{v}_{\mu}+{ }^{12} \mathrm{C}$ experiment presents real challenges to the theoretical models. Accordingly, one can expect rather divergent views about the impact of various reaction mechanisms.

\section{RESULTS}

Various studies have attempted to bring the predictions of (anti)neutrino-nucleus models in accordance with experimental data. Several modifications of the IA-based models have been considered, including the enhancement of the axial mass $M_{A}$ and the introduction of multinucleon effects $[16,19]$. These approaches have similar effects on neutrino scattering cross sections, bringing predictions closer to data. This impedes extraction of $M_{A}$ directly from the data and makes it difficult to use data to constrain the importance of multinucleon effects. In the following, we seek to shed light on these issues by making an analysis of QE cross sections and the relative importance of different contributions to neutrino and antineutrino scattering processes. We will show that multinucleon contributions and an enhanced axial mass affect the shape of the cross section differently and alter neutrino and antineutrino cross sections in a different way.

In order to test the robustness of calculations, we first investigate their sensitivity to the nuclear physics input. In computing the electroweak responses with the CRPA method, input is required with regard to the residual nucleon-nucleon interactions, the mean-field wave functions, and mean-field potential. In Fig. 3, the sensitivity of the computed cross sections
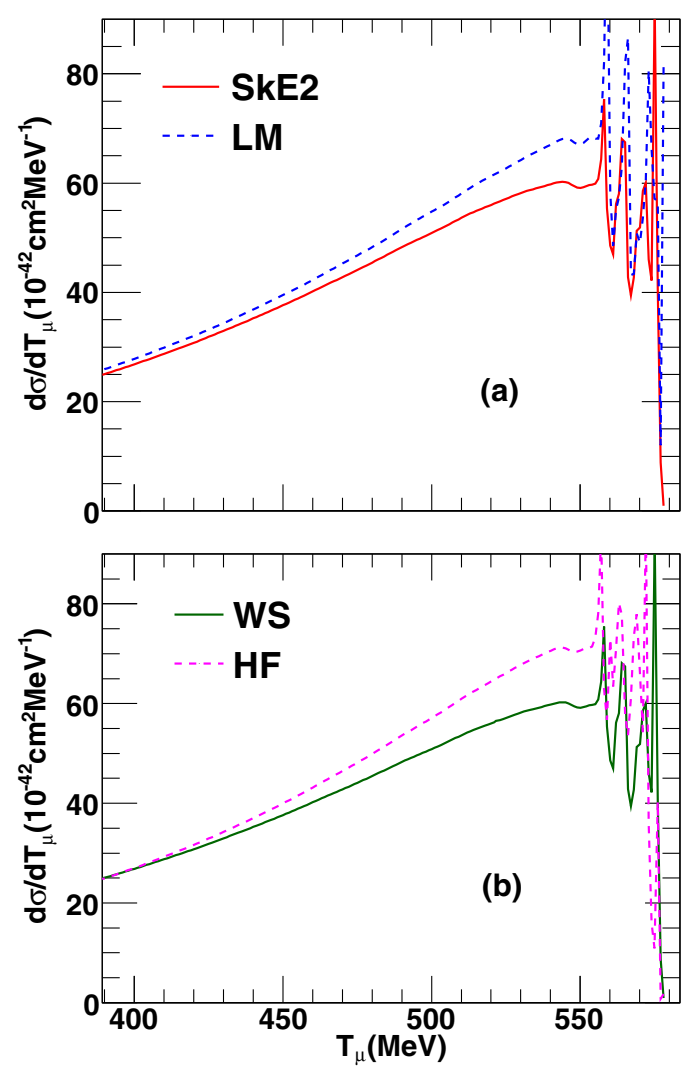

FIG. 3. (Color online) The $T_{\mu}$ dependence of the QE antineutrino- ${ }^{12} \mathrm{C}$ CRPA cross sections for $E_{\bar{v}_{\mu}}=700 \mathrm{MeV}$. (a) Curves obtained with the SkE2 and Landau-Migdal (LM) residual interaction with WS as single-particle wave functions. (b) Curves obtained with the WS and HF single-particle wave functions with $\mathrm{SkE} 2$ as residual interaction.

to the nuclear-physics input is studied at $E_{\bar{v}_{\mu}}=700 \mathrm{MeV}$. In the top panel, we compare cross sections obtained with a Skyrme (SkE2) [27,33] and a Landau-Migdal parametrization [34] for the residual effective nucleon-nucleon (NN) force. The sensitivity to the $\mathrm{NN}$ force is small for low outgoing muon energies but becomes substantial at higher $T_{\mu}$, corresponding to lower nuclear excitation energies where differences amount to almost $15 \%$. This is expected as it corresponds with a kinematic range most prone to nuclear collective effects. At low $T_{\mu}$, the cross sections are rather insensitive to the details of the residual $\mathrm{NN}$ force. A similar analysis is made for the use of different bound-state single-nucleon wave function Woods-Saxon (WS) [35] and Hartree-Fock (HF) in the bottom panel. Here again, significant differences up to $20 \%$ arise at higher $T_{\mu}$. Similar effects arise for calculations at other incoming energies. The strongest sensitivity, both for the shape and the magnitude of the cross section, to the nuclear-structure input occurs at the high- $T_{\mu}$ edges (corresponding to low nuclear excitation energies) of the computed cross sections. Concluding, even within the same approach, there is some sensitivity of the cross sections to the nuclear-structure input. We would like to stress that the parametrizations used in our calculations are not tuned in any way to the MiniBooNE data. 

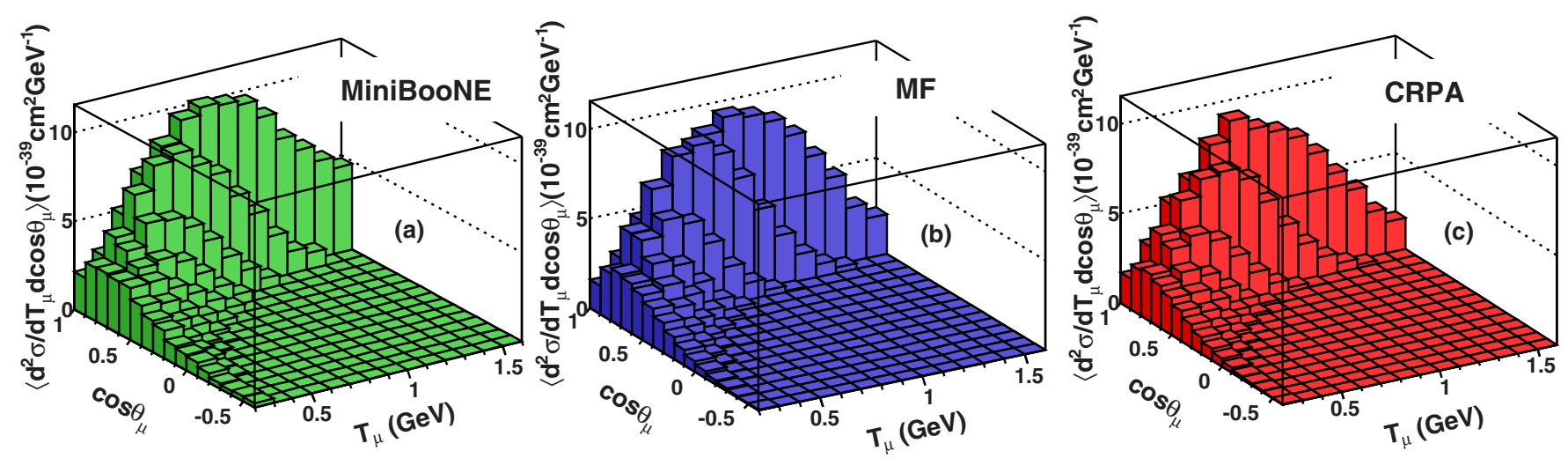

FIG. 4. (Color online) Double-differential cross section per target proton for ${ }^{12} \mathrm{C}\left(\bar{v}_{\mu}, \mu^{+}\right) X$, as a function of $T_{\mu}$ and $\cos \theta_{\mu}$. The MiniBooNE data [1] are plotted without the shape uncertainty and also excludes the $17.2 \%$ normalization uncertainty. CRPA and MF calculations are folded with MiniBooNE $\bar{v}_{\mu}$ flux.

The flux-integrated double-differential cross section for CCQE antineutrino-nucleus scattering, in terms of the measured quantities $T_{\mu}$ and $\cos \theta_{\mu}$ (hence free from the energy reconstruction issue $[9,23,36]$ ) can be written as

$$
\begin{aligned}
& \left\langle\frac{d^{2} \sigma}{d T_{\mu} d \cos \theta_{\mu}}\right\rangle \\
& \quad=\frac{1}{\int \Phi\left(E_{\bar{v}}\right) d E_{\bar{v}}} \int\left[\frac{d^{2} \sigma}{d T_{\mu} d \cos \theta_{\mu}}\right]_{E_{\bar{v}}} \Phi\left(E_{\bar{v}}\right) d E_{\bar{v}},
\end{aligned}
$$

where the antineutrino flux $\Phi\left(E_{\bar{v}}\right)$ is taken from [1]. The energy distribution of the MiniBooNE normalized antineutrino and neutrino flux is shown in Fig. 2. The neutrino flux peaks at higher energies than the antineutrino one.

In this work, incoming antineutrino energies up to $E_{\bar{v}_{\mu}}=$ $2 \mathrm{GeV}$ and multipoles up to $J=12$ are included in the calculations. We have checked that under all considered kinematic conditions, the computed inclusive antineutrino cross sections do not receive sizable contributions from $J>12$ multipoles. Unless specified otherwise, the used bound-state single-particle wave functions are solutions to the Schrödinger equation with a WS potential.

The double-differential ${ }^{12} \mathrm{C}\left(\bar{v}_{\mu}, \mu^{+}\right) X$ cross sections per target proton are displayed in Fig. 4. The CRPA and MF calculations are folded with the MiniBooNE $\bar{v}_{\mu}$ flux of Fig. 2. In the dipole axial form factor, we adopt $M_{A}=1.03 \mathrm{GeV}$ which is essentially tuned to deuterium bubble-chamber data. The uncertainties (both with regard to shape and to normalization) in the MiniBooNE data are not shown. Comparing the CRPA and MF results in Fig. 4, it is clear that the inclusion of RPA correlations reduces the cross sections, at the same time shifting the strength towards lower muon energies. Obviously, both the MF and CRPA calculations reproduce the major features of the measured $\left(\cos \theta_{\mu}, T_{\mu}\right)$ distributions: the largest cross sections are for forward $\theta_{\mu}$ and the peaks shift to smaller $T_{\mu}$ with increasing $\theta_{\mu}$. This is completely in line with the expectations from the $\left(\cos \theta_{\mu}, T_{\mu}\right)$ dependence of the $x_{B}$ and minimum $p_{\text {mis }}$ of Fig. 1 .

Figure 5 shows a more detailed picture, displaying doubledifferential cross sections as a function of $T_{\mu}\left(\cos \theta_{\mu}\right)$ for various bins in the other kinematic variable. The theoretical results are obtained by integrating the calculations over the corresponding bin width. The MiniBooNE data of Fig. 5 include the experimental uncertainties. Overall, the CRPA predictions are in satisfactory agreement with the data. The quality of agreement between the CRPA calculations and data is best at low and average muon kinetic energies and forward muon angles. At backward $\cos \theta_{\mu}$, the CRPA tends to underestimate the data for higher- $T_{\mu}$ values. It has been suggested by several authors that multinucleon excitations are at the origin of the missing strength at higher $T_{\mu}$ and backward $\theta_{\mu}$, as that region corresponds with large values of $x_{B}$ and minimum $p_{\text {mis }}$. The quenching due to RPA correlations is strongest at backward $\theta_{\mu}$ and disappears at the $T_{\mu}$ edges of the distributions. In general, the MF provides a better description of the data than CRPA both for the shape and magnitude of the cross section. A similar observation was made in Ref. [37], where two approaches are considered to compute the CCQE $v_{\mu}+{ }^{12} \mathrm{C}$ cross sections, superscaling, and the relativistic mean-field (RMF) approach. Of these two, the RMF model was observed to provide the best description of the shape of the double-differential cross sections. Our calculations are in line with those of the RMF model of [13], yet slightly closer to the data.

Various studies have observed different contributions of RPA and multinucleon effects for neutrino and antineutrino cross sections. The top panel of Fig. 6 shows QE neutrino and antineutrino cross sections, both normalized to one. In absolute numbers, the neutrino cross section is always larger, but the normalized cross section shows that antineutrino processes exhibit a stronger sensitivity to contributions stemming from the high end of the $T_{\mu}$ spectrum. As illustrated in the bottom panel of Fig. 6, this difference can be explained by the sign of the transverse interference term in Eq. (6). For neutrinos, both transverse terms add constructively, while for antineutrinos they add destructively. The absolute value of the interference contribution to $\sigma_{T}$ is relatively small. Still, for low $T_{\mu}$, the comparable size of both transverse contributions results in a sizable gain of importance of the transverse interference term. Therefore, at low $T_{\mu}$, the difference between the $v_{\mu}$ and $\bar{v}_{\mu}$ cross sections increases and the antineutrino ones become very small. Hence, the main contribution to antineutrino 

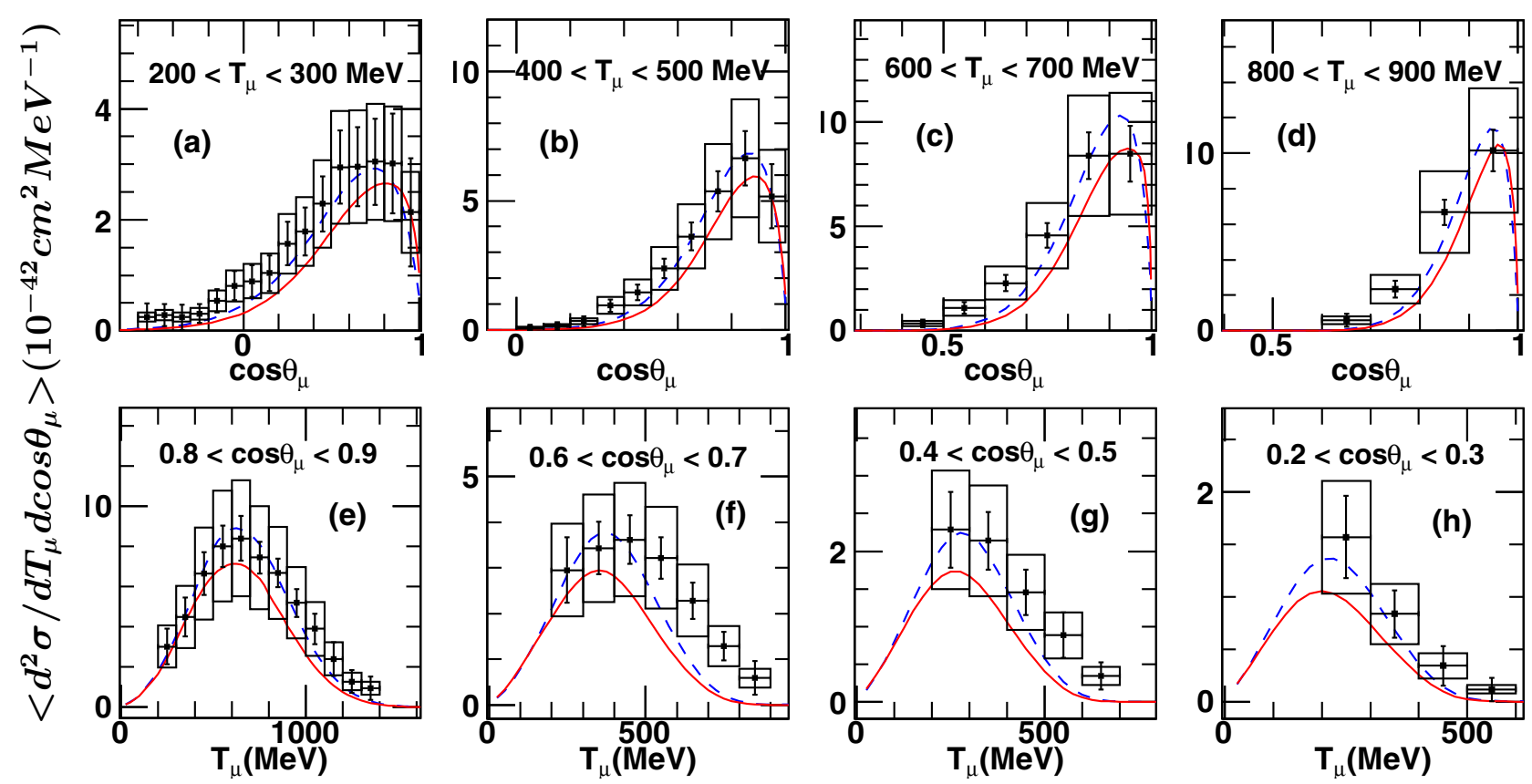

FIG. 5. (Color online) MiniBooNE flux-folded double-differential cross section per target proton for ${ }^{12} \mathrm{C}\left(\bar{v}_{\mu}, \mu^{+}\right) X$ plotted as a function $T_{\mu}$ for different ranges of $\cos \theta_{\mu}$ (bottom), as a function of $\cos \theta_{\mu}$ for different $T_{\mu}$ values (top). Solid curves are CRPA and dashed curves are MF calculations. MiniBooNE data are filled squares, error bars represent the shape uncertainties, and error boxes represent the $17.2 \%$ normalization uncertainty.

scattering comes from reactions at higher- $T_{\mu}$ values and antineutrino-nucleus reactions are relatively more sensitive to low-energy nuclear dynamics than their neutrino counterparts. As can be appreciated from Fig. 6, low nuclear excitation energies represent a large share of the folded cross sections. Accordingly, one may expect that the effect of the RPA correlations is stronger for $\bar{v}_{\mu} A$ interactions.

As a consequence of these differences and the respective energy dependence of cross sections, one can also expect differing influences of multinucleon effects on neutrino and antineutrino cross sections. The effect of multinucleon contributions to the $\bar{v}_{\mu}$ double-differential cross sections is studied among others in Refs. $[11,12,14]$ and to the $v_{\mu}$ cross sections in Ref. [17,37,38]. From those studies, particularly from Figs. 1 and 4 in Ref. [37], it emerges that for the very forward-peaked neutrino scattering in MiniBooNE kinematics, multinucleon contributions are responsible for a significant fraction of the strength at low $T_{\mu}$ and are essential for reproducing the data. At backward $\theta_{\mu}$, where cross sections are very small anyway, the effect of the multinucleon contributions is rather modest. This can be understood by realizing that backward $\theta_{\mu}$ corresponds with larger values of $Q^{2}$ (Fig. 1). With increasing values of the range parameter $Q^{2}$, multinucleon effects naturally lose in importance [39]. In the superscaling approach of Ref. [37], it is argued that the relative impact of $n \mathrm{p}-n \mathrm{~h}$ contributions increases with growing energies of the incoming lepton. Moreover, pionless intermediate $\Delta$ creation is a source of strength beyond the IA that gains in importance as one approaches the pole of the $\Delta$ propagator $[39,40]$. From Fig. 6 it became obvious that the antineutrino-nucleus reaction has an enhanced sensitivity to the strength stemming from lower nuclear excitation energies. More specifically in the MiniBooNE experiment, the antineutrino flux peaks at lower energies than the neutrino one as shown in Fig. 2. Under those kinematic circumstances, one might expect strong nuclear effects but reduced $n \mathrm{p}-n \mathrm{~h}$ contributions through pionless $\Delta$ decay, for example.

Obviously, modeling the multitude of $n \mathrm{p}-n \mathrm{~h}$ effects at various energies introduces uncertainties. Figure 7 shows the predicted contribution from $n \mathrm{p}-n \mathrm{~h}$ to the ${ }^{12} \mathrm{C}\left(\bar{v}_{\mu}, \mu^{+}\right) X$ cross section for two models available in the literature. Whereas the shape of the energy dependence of the multinucleon contribution is predicted slightly differently in these studies, its magnitude differs considerably. In both studies, the shape of flux-averaged $n \mathrm{p}-n \mathrm{~h}$ contributions is similar to that of the $\mathrm{QE}$ cross section. The divergent views about the role of the $n \mathrm{p}-n \mathrm{~h}$ illustrate that the model dependencies are unavoidable given the extensive range of $x_{B}, p_{\mathrm{mis}}^{\mathrm{min}}, Q^{2}$ (Fig. 1) values covered in the experiments. The good general agreement of the calculations is mainly obtained by the combination of QE and multinucleon contributions, averaging out the most apparent discrepancies.

The (anti)neutrino-nucleus response calculations require input with regard to the two vector and the axial form factors. They are often parametrized as a dipole function of the range parameter $Q^{2}$. As a result, each form factor introduces at least two parameters, a cutoff mass, formally playing the role of a size parameter and the value at $Q^{2}=0$ that determines the coupling strength. The two vector form factors are well known from electron-scattering studies [41] and we use a standard dipole parametrization which is a good approximation for the $Q^{2}$ values probed in MiniBooNE (Fig. 1). The axial form 

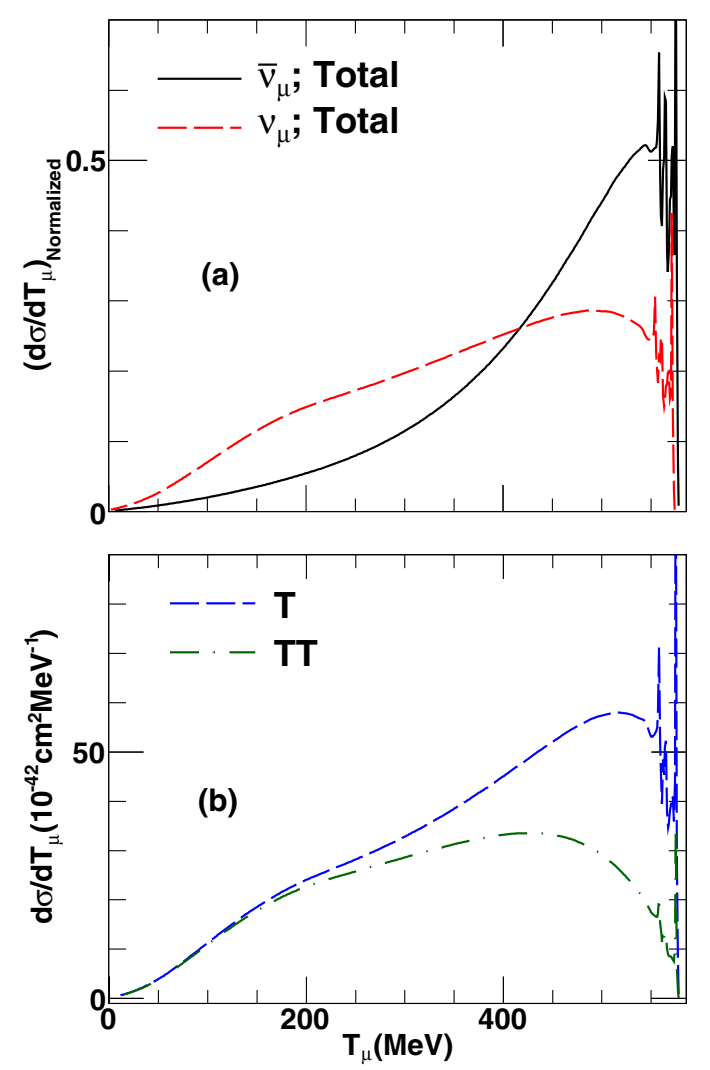

FIG. 6. (Color online) The $T_{\mu}$ distribution of the CRPA ${ }^{12} \mathrm{C}\left(\bar{v}_{\mu}, \mu^{+}\right)$and ${ }^{12} \mathrm{C}\left(v_{\mu}, \mu^{-}\right)$cross sections at a (anti)neutrino energy of $700 \mathrm{MeV}$. (a) Total cross sections normalized to 1. (b) Transverse contribution excluding the interference part $(\mathrm{T})$ and the transverse interference (TT) contribution.

factor, in the dipole form, reads as

$$
G_{A}=\frac{g_{A}}{\left(1+\frac{Q^{2}}{M_{A}^{2}}\right)^{2}},
$$

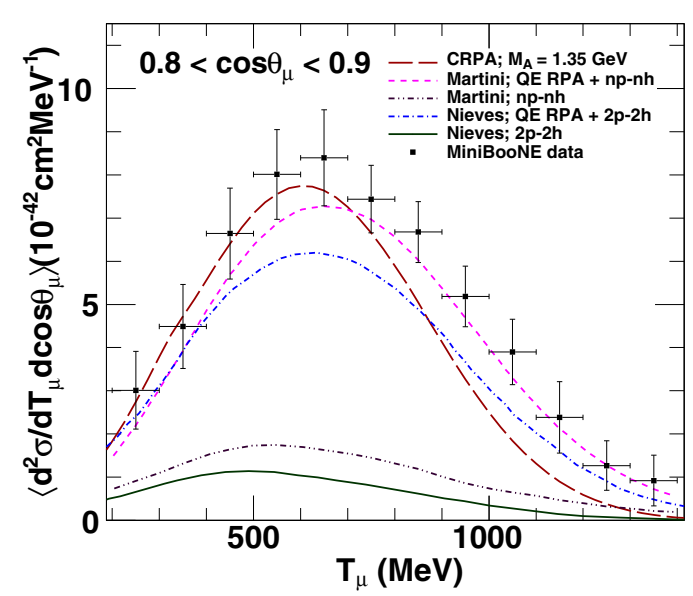

FIG. 7. (Color online) MiniBooNE flux-folded cross section per target proton for ${ }^{12} \mathrm{C}\left(\bar{v}_{\mu}, \mu^{+}\right) X$ at $0.8<\cos \theta_{\mu}<0.9$. The CRPA predictions are compared with those of Refs. [11,12].
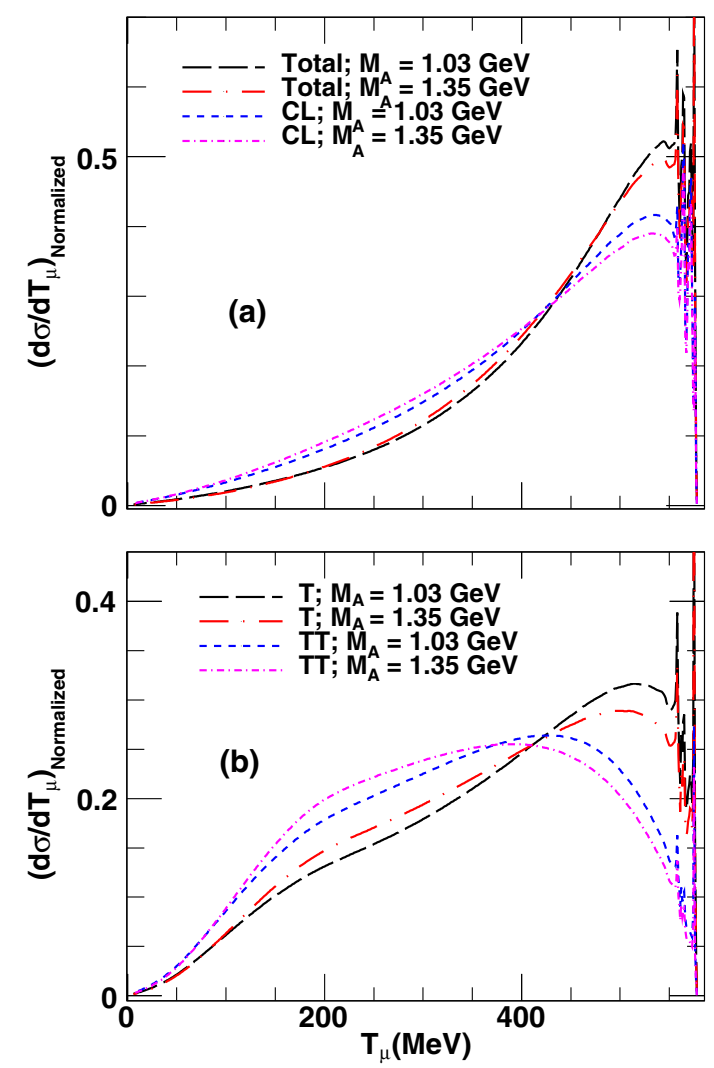

FIG. 8. (Color online) Normalized Coulomb longitudinal (CL), transverse term without interference $(\mathrm{T})$, and transverse interference (TT) contributions to ${ }^{12} \mathrm{C}\left(\bar{v}_{\mu}, \mu^{+}\right) X$ as a function of $T_{\mu}$ for two different values of $M_{A}$, at $E_{\bar{v}_{\mu}}=700 \mathrm{MeV}$.

where $g_{A}$ is determined from nuclear $\beta$ decay [42]. The value $M_{A}=1.03 \pm 0.02 \mathrm{GeV}$ is regarded as the world's average value [43-45] emerging from bubble-chamber experiments. Tuning Eq. (10) to the shape of the $Q^{2}$ distribution of the MiniBooNE $v_{\mu}$ data $[1,3]$ favors the value $M_{A}=1.35 \pm 0.17 \mathrm{GeV}$. In Fig. 8, we investigate the sensitivity of the computed CRPA cross sections to the adopted value of $M_{A}$. Changing $M_{A}$ from 1.03 to $1.35 \mathrm{GeV}$ increases the cross sections by nearly $10 \%$. Note that in Fig. 8 we present the normalized cross sections. From the figure, it can be appreciated that modification of $M_{A}$ affects both the energy distribution and the $\sigma_{C L} / \sigma_{T}$ ratio. Whereas the overall effect of enhancing $M_{A}$ is a cross-section increase, this figure shows that more subtle mechanisms are at play. Enhancing $M_{A}$ shifts the strength to higher nuclear excitation energies, resulting in a larger impact on the MiniBooNE neutrino than antineutrino cross sections.

In Fig. 9, we study the sensitivity of the double-differential flux-folded CRPA cross section to the adopted value of $M_{A}$. It can be appreciated that enhancing $M_{A}$ improves the overall agreement between the CRPA antineutrino cross sections and the data. The enhancement is most pronounced at backward muon scattering but still does not suffice to bring calculations in agreement with data, especially for higher $T_{\mu}$. As becomes clear from Fig. 10, with $M_{A}=1.35 \mathrm{GeV}$, the CRPA results reproduce the data for $T_{\mu} \leqslant 600 \mathrm{MeV}$ well. Under those 

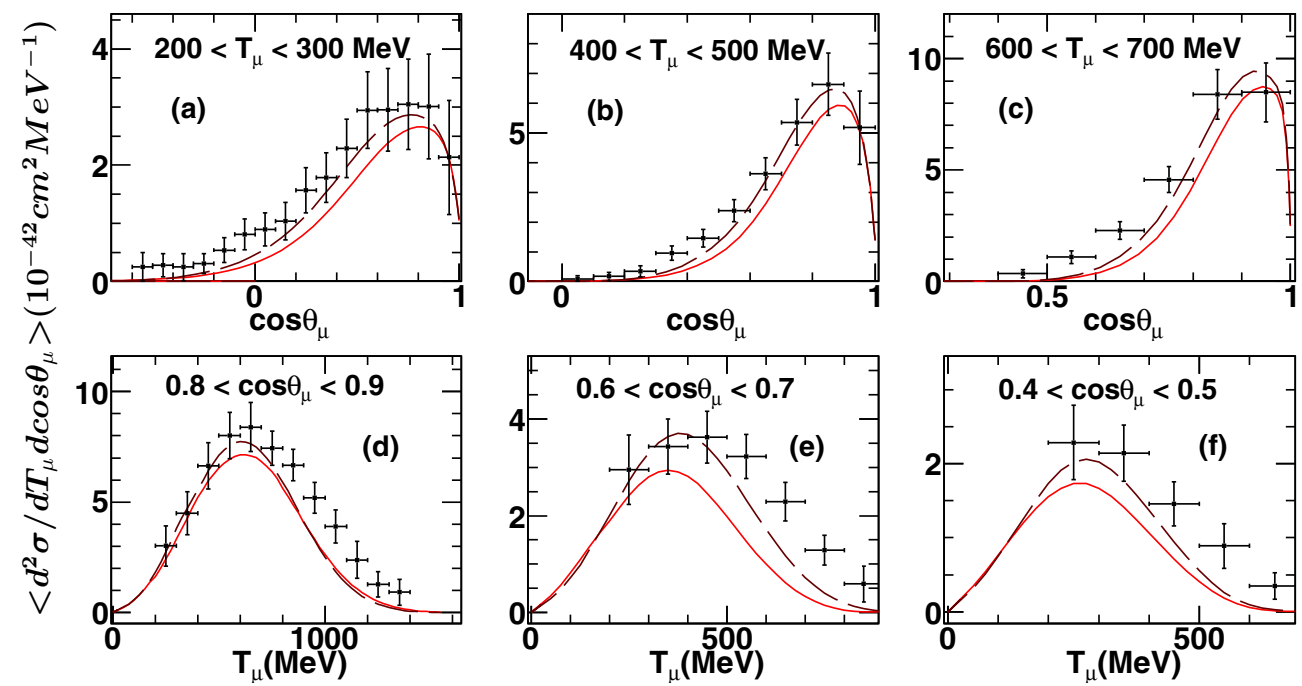

FIG. 9. (Color online) MiniBooNE flux-folded double-differential cross section per target proton for ${ }^{12} \mathrm{C}\left(\bar{v}_{\mu}, \mu^{+}\right) X$. Full (dashed) curves are CRPA with $M_{A}=1.03 \mathrm{GeV}\left(M_{A}=1.35 \mathrm{GeV}\right)$. MiniBooNE data are filled squares shown with shape uncertainty which excludes an additional $17.2 \%$ normalization uncertainty. The top panels show the $\cos \theta_{\mu}$ dependence for different ranges of $T_{\mu}$, while the lower panels give the $T_{\mu}$ dependence for different ranges of $\cos \theta_{\mu}$.

kinematic conditions, the calculations of Ref. [11] tend to underestimate the data. At higher values of $T_{\mu}$ the opposite situation occurs with CRPA underestimating the data. From
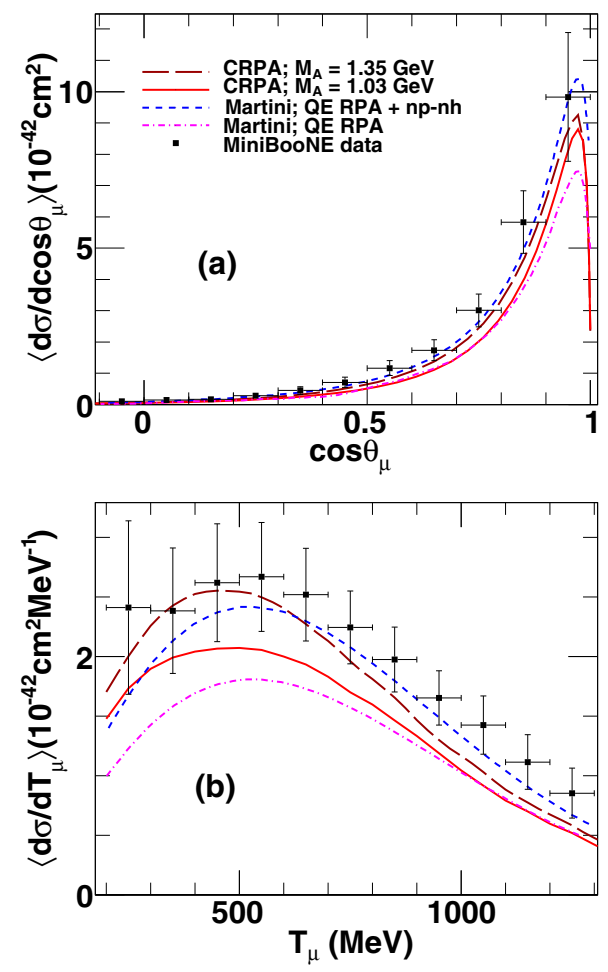

FIG. 10. (Color online) MiniBooNE flux-folded cross section per target proton for ${ }^{12} \mathrm{C}\left(\bar{v}_{\mu}, \mu^{+}\right) X$ as a function of $\cos \theta_{\mu}$ (a) and of $T_{\mu}$ (b). A comparison is made of the CRPA cross sections with those of Ref. [11] (Martini et al.). The MiniBooNE data are integrated over $T_{\mu}$ (a) and over $\cos \theta_{\mu}$ (b). the comparison in Fig. 10, we also find that our CRPA cross sections are larger than the QE RPA predictions from Ref. [11].

The analysis of the MINERvA antineutrino results [7] favors the transverse enhancement model (TEM). In TEM, the magnetic form factors of the bound nucleons are modified in order to account for the enhancement relative to IA predictions, observed in the transverse parts of the electron-nucleus cross sections [46]. We stress that in the analysis of Ref. [7], the TEM and $M_{A}=1.35 \mathrm{GeV}$ models predict comparable cross sections at $Q^{2} \lesssim 1 \mathrm{GeV}^{2}$. Accordingly, one can anticipate that for the $Q^{2}$ region accessible at MiniBooNE energies (Fig. 1), it is difficult to discriminate between the two effective ways of enhancing the computed weak responses.

\section{CONCLUSIONS}

We have calculated the MiniBooNE flux-folded QE contribution to the ${ }^{12} \mathrm{C}$-antineutrino cross sections and present the results in terms of the experimentally measured quantities $T_{\mu}$ and $\cos \theta_{\mu}$. The predictions are made within a nonrelativistic CRPA. The overall agreement between our predictions for the QE contribution to antineutrino scattering cross sections and the MiniBooNE measurements is satisfactory. The best description is reached for lower $T_{\mu}$. At higher muon kinetic energies and backward scattering angles, the CRPA results underestimate the data. At larger $T_{\mu}$, one observes a significant sensitivity to the choices made with regard to the nucleonnucleon interaction and the single-particle wave functions. We observe that the mean-field cross sections in our calculations are in line with the results of [13] and larger than those of Fermi-gas calculations.

As antineutrino cross sections are more sensitive to lowenergy nuclear dynamics, an effect that becomes even more pronounced owing to energy distribution of the MiniBooNE antineutrino flux, the effect of RPA correlations is stronger for antineutrinos than for neutrinos. For the MiniBooNE 
kinematic regime and the very forward scattering dominated neutrino interactions, multinucleon mechanism can be expected to be most important for reactions with a low-energy outgoing lepton. Enhancing $M_{A}$ enhances the cross sections mostly at higher $T_{\mu}$ and backward scattering angles. Altering $M_{A}$ has a larger influence on neutrino than on antineutrino cross sections. Still, we observe that in case of antineutrino scattering at MiniBooNE energies, an enhancement in the nucleon axial mass seems to be an effective way of improving the quality of agreement between the CRPA calculations and the data not only for the size, but also for the shape of the double-differential cross section.

\section{ACKNOWLEDGMENT}

This research was funded by the Interuniversity Attraction Poles Programme initiated by the Belgian Science Policy Office, the Erasmus Mundus External Cooperations Window's Eurindia Project, and the Research Foundation Flanders (FWO-Flanders).
[1] A. A. Aguilar-Arevalo et al. (MiniBooNE Collaboration), Phys. Rev. D 88, 032001 (2013).

[2] J. A. Formaggio and G. P. Zeller, Rev. Mod. Phys. 84, 1307 (2012).

[3] A. A. Aguilar-Arevalo et al. (MiniBooNE Collaboration), Phys. Rev. D 81, 092005 (2010).

[4] A. A. Aguilar-Arevalo et al. (MiniBooNE Collaboration), Phys. Rev. D 82, 092005 (2010).

[5] K. Abe et al. (T2K Collaboration), Phys. Rev. D 87, 092003 (2013).

[6] G. A. Fiorentini et al. (MINERvA Collaboration), Phys. Rev. Lett. 111, 022502 (2013).

[7] L. Fields et al. (MINERvA Collaboration), Phys. Rev. Lett. 111, 022501 (2013).

[8] J. G. Morfin, J. Nieves, and J. T. Sobczyk, Adv. High Energy Phys. 2012, 934597 (2012).

[9] O. Lalakulich, U. Mosel, and K. Gallmeister, Phys. Rev. C 86, 054606 (2012).

[10] O. Benhar, P. Coletti, and D. Meloni, Phys. Rev. Lett. 105, 132301 (2010).

[11] M. Martini and M. Ericson, Phys. Rev. C 87, 065501 (2013).

[12] J. Nieves, I. Ruiz Simo, and M. J. Vicente Vacas, Phys. Lett. B 721, 90 (2013).

[13] M. V. Ivanov, R. González-Jiménez, J. A. Caballero, M. B. Barbaro, T. W. Donnelly, and J. M. Udías, Phys. Lett. B 727, 265 (2013).

[14] J. E. Amaro, M. B. Barbaro, J. A. Caballero, and T. W. Donnelly, Phys. Rev. Lett. 108, 152501 (2012).

[15] A. Meucci and C. Giusti, Phys. Rev. D 85, 093002 (2012).

[16] M. Martini, M. Ericson, G. Chanfray, and J. Marteau, Phys. Rev. C 80, 065501 (2009).

[17] M. Martini, M. Ericson, and G. Chanfray, Phys. Rev. C 84, 055502 (2011).

[18] M. Martini, M. Ericson, G. Chanfray, and J. Marteau, Phys. Rev. C 81, 045502 (2010).

[19] J. Nieves, I. R. Simo, and M. J. Vicente Vacas, Phys. Rev. C 83, 045501 (2011).

[20] G. Shen, L. E. Marcucci, J. Carlson, S. Gandolfi, and R. Schiavilla, Phys. Rev. C 86, 035503 (2012).

[21] J. E. Amaro, M. B. Barbaro, J. A. Caballero, T. W. Donnelly, and C. Maieron, Phys. Rev. C 71, 065501 (2005).

[22] J. E. Amaro, M. B. Barbaro, J. A. Caballero, T. W. Donnelly, and J. M. Udias, Phys. Rev. C 75, 034613 (2007).
[23] J. Nieves, F. Sanchez, I. R. Simo, and M. J. Vicente Vacas, Phys. Rev. D 85, 113008 (2012).

[24] N. Jachowicz, S. Rombouts, K. Heyde, and J. Ryckebusch, Phys. Rev. C 59, 3246 (1999).

[25] N. Jachowicz, K. Heyde, J. Ryckebusch, and S. Rombouts, Phys. Rev. C 65, 025501 (2002).

[26] J. Ryckebusch, M. Waroquier, K. Heyde, J. Moreau, and D. Ryckbosch, Nucl. Phys. A 476, 237 (1988).

[27] J. Ryckebusch, K. Heyde, D. Van Neck, and M. Waroquier, Nucl. Phys. A 503, 694 (1989).

[28] N. Jachowicz, C. Praet, and J. Ryckebusch, Acta Phys. Pol., B 40, 2559 (2009).

[29] N. Jachowicz and V. Pandey (unpublished).

[30] J. E. Amaro, M. B. Barbaro, J. A. Caballero, and T. W. Donnelly, Phys. Rev. C 73, 035503 (2006).

[31] M. Vanhalst, J. Ryckebusch, and W. Cosyn, Phys. Rev. C 86, 044619 (2012).

[32] S. Janssen, J. Ryckebusch, W. Van Nespen, and D. Debruyne, Nucl. Phys. A 672, 285 (2000).

[33] M. Waroquier, J. Ryckebusch, J. Moreau, K. Heyde, N. Blasi, S. Y. van de Werf, and G. Wenes, Phys. Rep. 148, 249 (1987).

[34] G. Có and S. Krewald, Nucl. Phys. A 433, 392 (1985).

[35] G. Có and S. Krewald, Phys. Lett. B 137, 145 (1984).

[36] M. Martini, M. Ericson, and G. Chanfray, Phys. Rev. D 85, 093012 (2012).

[37] J. E. Amaro, M. B. Barbaro, J. A. Caballero, T. W. Donnelly, and J. M. Udias, Phys. Rev. D 84, 033004 (2011).

[38] J. Nieves, I. Ruiz Simo, and M. J. Vicente Vacas, Phys. Lett. B 707, 72 (2012).

[39] J. Ryckebusch, D. Debruyne, W. Van Nespen, and S. Janssen, Phys. Rev. C 60, 034604 (1999).

[40] J. Ryckebusch, Phys. Rev. C 64, 044606 (2001).

[41] H. Budd, A. Bodek, and J. Arrington, Nucl. Phys. B 139, 90 (2005).

[42] J. Beringer et al. (Particle Data Group), Phys. Rev. D 86, 010001 (2012).

[43] C. Amsler et al. (Particle Data Group), Phys. Lett. B 667, 1 (2008)

[44] V. Bernard, L. Elouadrhiri, and U. G. Meissner, J. Phys. G: Nucl. Part. Phys. 28, R1 (2002).

[45] V. Lyubushkin et al. (NOMAD Collaboration), Eur. Phys. J. C 63, 355 (2009).

[46] A. Bodek, H. S. Budd, and M. E. Christy, Eur. Phys. J. C 71, 1726 (2011). 